\title{
Imaging the relationship between structure, function and behaviour in the human brain
}

\author{
Heidi Johansen-Berg
}

Received: 29 July 2009/Accepted: 27 August 2009/Published online: 10 September 2009

(C) Springer-Verlag 2009

Neuroscience has its roots in the study of brain anatomy. For centuries, scientists studied the gross structure of the brain and, as technology developed, went on to perform careful studies of the microstructure of grey matter and the course of white matter fibres (Finger 1994; Schmahmann and Pandya 2007). However, the development of modern day techniques for functional neuroimaging arguably led to neglect of brain structure in favour of a focus on mapping functional responses associated with performance of specific behaviours.

Yet brain anatomy and brain function are inextricably linked and together determine our behavioural responses. The cytoarchitecture of a region tells us about the cell types present, which dictates the processing operations that can be computed. The distribution of receptors within a region will determine the ways in which neurochemistry can modulate its operation (Zilles et al. 1995; Zilles and Amunts 2009). The anatomical inputs to a region determine the information available to it while its outputs dictate the influence that one area can have over other regions of the brain (Passingham et al. 2002). The structural features of these white matter connections, such as their myelination and axon diameter, will determine physiological properties such as conduction velocity and refractory time (Fields 2008).

In recent years, neuroimaging approaches for probing the gross architecture and the microstructural anatomy of the brain have reinvigorated the study of human brain

H. Johansen-Berg $(\bowtie)$

Oxford Centre for Functional MRI of the Brain,

University of Oxford, Oxford OX3 9DU, UK

e-mail: heidi@fmrib.ox.ac.uk anatomy and are now increasingly used in combination with functional imaging techniques (Bandettini 2009). For example, variations in sulcal and gyral anatomy or in cortical thickness can be objectively and quantitatively investigated (Riviere et al. 2002; Dale et al. 1999; Fischl et al. 1999; Van Essen 2005). Localised differences in imaging contrast of the grey matter, influenced by factors such as myeloarchitecture (Eickhoff et al. 2005), cytoarchitecture and cortical thickness, can be objectively quantified using whole brain statistical techniques such as voxel-based morphometry (Ashburner 2009; Ridgway et al. 2008). Diffusion imaging provides measures of white matter microstructure that are influenced by features such as axon diameter, packing density and myelination (Beaulieu 2009) and can be used to perform non-invasive tractography (Jones et al. 1999; Behrens et al. 2003), allowing for in vivo estimates of major fibre trajectories.

Compared with 'gold-standard' techniques for brain anatomy, such as histological techniques or tracer-based tract tracing, the neuroimaging approaches are crude and difficult to interpret. There is rarely a one-to-one relationship between a given imaging measure and an underlying anatomical feature. The emerging techniques will benefit from careful validation and ongoing development but it is likely that neuroimaging studies of brain anatomy will always rely on information from classical neuroanatomy to guide their interpretation. Nevertheless, an enormous advantage of neuroimaging-based studies of brain structure is that they can be performed in living human brains whose functional responses and behavioural outputs can be studied at the same time. This offers unparalleled opportunities for studying whole brain anatomy in health and disease. This special issue brings together papers using neuroimaging to address the relationships between human brain structure, function and behaviour. 


\section{References}

Ashburner J (2009) Computational anatomy with the SPM software. Magn Reson Imaging

Bandettini PA (2009) What's new in neuroimaging methods? Ann N Y Acad Sci 1156:260-293

Beaulieu C (2009) The biological basis of diffusion anisotropy. In: Johansen-Berg H, Behrens TEJ (eds) Diffusion MRI: from quantitative measurement to in vivo neuroanatomy. Elsevier, London

Behrens TEJ et al (2003) Characterization and propagation of uncertainty in diffusion-weighted MR imaging. Magn Reson Med 50:1077-1088

Dale AM, Fischl B, Sereno MI (1999) Cortical surface-based analysis. I. Segmentation and surface reconstruction. NeuroImage 9:179-194

Eickhoff S et al (2005) High-resolution MRI reflects myeloarchitecture and cytoarchitecture of human cerebral cortex. Hum Brain Mapp 24:206-215

Fields RD (2008) White matter in learning, cognition and psychiatric disorders. Trends Neurosci 31:361-370

Finger S (1994) Origins of neuroscience. Oxford University Press, New York

Fischl B, Sereno MI, Dale AM (1999) Cortical surface-based analysis. II: inflation, flattening, and a surface-based coordinate system. NeuroImage 9:195-207
Jones DK, Simmons A, Williams SC, Horsfield MA (1999) Noninvasive assessment of axonal fiber connectivity in the human brain via diffusion tensor MRI. Magn Reson Med 42:37

Passingham RE, Stephan KE, Kotter R (2002) The anatomical basis of functional localization in the cortex. Nat Rev Neurosci 3:606

Ridgway GR et al (2008) Ten simple rules for reporting voxel-based morphometry studies. NeuroImage 40:1429-1435

Riviere D et al (2002) Automatic recognition of cortical sulci of the human brain using a congregation of neural networks. Med Image Anal 6:77-92

Schmahmann JD, Pandya DN (2007) Cerebral white matter-historical evolution of facts and notions concerning the organization of the fiber pathways of the brain. J Hist Neurosci 16:237-267

Van Essen DC (2005) A population-average, landmark- and surfacebased (PALS) atlas of human cerebral cortex. NeuroImage 28:635-662

Zilles K, Amunts K (2009) Receptor mapping: architecture of the human cerebral cortex. Curr Opin Neurol 22:331-339

Zilles K et al (1995) Mapping of human and macaque sensorimotor areas by integrating architectonic, transmitter receptor, MRI and PET data. J Anat 187:515 